\title{
Incidencia de tromboembolismo venoso en cirugía ortognática
}

\author{
Andrés Campolo G., Tomás Rioseco I., Ignacio Goñi E., \\ Alex Vargas D. y Hernán Ramírez S.
}

Facultad de Medicina. Pontificia Universidad Católica de Chile.

Recibido el 16 de noviembre de 2017, aceptado para publicación el

7 de marzo de 2018

Correspondencia a: Andrés Campolo G. andres.campolo@gmail.com

\section{Incidence of venous thromboembolism in orthognathic surgery}

Introduction: Venous thromboembolism (VTE) is an important cause of morbidity and mortality in surgical patients. This entity has well defined and described pathophysiological mechanisms, in addition to risk factors that allow a classification of patients according to different assessment models, which allow to adopt prophylactic measures. In maxillofacial surgery, low levels of incidence have been described. However, there is quite limited evidence and represents a health problem that has received little attention in the literature. Objective: To determine the incidence of venous thromboembolism in patients undergoing orthognathic surgery. Material and Method: Retrospectively, 86 patients who underwent orthognathic surgery was evaluated between October 2006 and January 2016 at the Hospital Clínico de la Pontificia Universidad Católica de Chile. Two cases of pulmonary thromboembolism were identified, which corresponds to an incidence of $2.6 \%$. Despite the low incidence of VTE in patients undergoing maxillofacial surgery, it is essential to consider the individual risk of the patient and the risk involved in surgery. The prolongation of surgical time, the hospital stay and prolonged immobilization can determine a greater risk of developing this type of complication, therefore it is necessary to consider it and know how to prevent it in an adequate way.

Key words: venous thromboembolism; orthognathic surgery.

\section{Resumen}

Introducción: El tromboembolismo venoso (TEV) es una causa importante de morbimortalidad en la población hospitalaria y quirúrgica. Esta entidad tiene mecanismos fisiopatológicos bien definidos y descritos, además, de factores de riesgo que permiten una clasificación de los pacientes según diversos modelos de valoración, los cuales permiten adoptar medidas profilácticas. En el ámbito de la cirugía maxilofacial se han descrito bajos niveles de incidencia. Sin embargo, hay evidencia bastante limitada y representa un problema de salud al cual se le ha prestado poca atención en la literatura. Objetivo: El presente estudio tiene como objetivo conocer la incidencia de tromboembolismo venoso en pacientes sometidos a cirugía ortognática. Material y Método: Se evaluaron en forma retrospectiva fichas clínicas de 86 pacientes operados de cirugía ortognática entre octubre de 2006 y enero de 2016 en el Hospital Clínico de la Pontificia Universidad Católica de Chile. Se identificaron 2 casos de tromboembolismo pulmonar, lo cual corresponde a una incidencia de $2,6 \%$. Sin embargo, no se obtuvo un espacio muestral suficiente para lograr identificar factores de riesgo específicos en esta población. Conclusiones: A pesar de la baja incidencia de TEV en pacientes sometidos a cirugía maxilofacial, es fundamental considerar el riesgo individual del paciente y el riesgo que implica la cirugía. La prolongación del tiempo quirúrgico, la estadía hospitalaria y la inmovilización prolongada pueden determinar un riesgo mayor de desarrollar este tipo de complicación, por lo tanto, es necesario considerarla y saber prevenirla de manera adecuada.

Palabras clave: tromboembolismo venoso; cirugía ortognática. 


\section{Introducción}

El tromboembolismo venoso (TEV) comprende a dos entidades clínicas relacionadas: la trombosis venosa profunda (TVP) y el tromboembolismo pulmonar $(\mathrm{TEP})^{1}$, y es una complicación relativamente común asociada a procedimientos quirúrgicos, trauma o inmovilización prolongada. Además, puede llevar a mayores complicaciones como el síndrome postrombótico, hipertensión pulmonar y aumento en la mortalidad ${ }^{2}$.

En 1884, Rudolph Virchow fue el primero en describir el mecanismo fisiopatológico por el cual se produce el tromboembolismo venoso, planteando que la trombosis es el resultado de al menos uno de tres factores etiológicos; daño endotelial vascular, estasia venosa e hipercoagulabilidad. En el transcurso del último siglo se han reconocido distintos factores de riesgo que reflejan alguno de estos procesos fisiopatológicos, en ausencia de los cuales no suele desarrollarse el TEV ${ }^{1}$.

Los factores que han demostrado aumentar el riesgo de TEV son la edad avanzada, inmovilización prolongada, tumores malignos, cirugía mayor, trauma múltiple, TEV previo e insuficiencia cardiaca. Sin embargo, es importante reconocer que el valor predictivo de dichos factores no es idéntico. Para determinar adecuadamente si se requiere de profilaxis, el médico debe considerar tanto el peso individual de cada uno de los factores como la suma de ellos ${ }^{1}$.

Varios estudios en cirugía general han mostrado que el riesgo de desarrollar TVP es menor al 3\% para pacientes bajo los 40 años de edad y en aquellos que se someten a cirugías que duren menos de $30 \mathrm{~min}$. El riesgo aumenta acorde a la edad, la complejidad y la duración de la cirugía. Es mayor aún en pacientes obesos, pacientes con historia de TEV y en pacientes con tumores malignos. Otros factores de riesgo son la inmovilización prolongada, el uso de anticonceptivos orales, trauma múltiple y la insuficiencia cardiaca ${ }^{1,3,4}$.
En la actualidad, existe evidencia limitada respecto a la incidencia y factores de riesgo potenciales de TVP y TEP en pacientes que se someten a una cirugía maxilofacial. Es por ello que, el uso de tromboprofilaxis en pacientes que se someten a dicho tipo de procedimientos, no ha recibido mucha atención.

El presente estudio tiene como objetivo analizar retrospectivamente la incidencia de TVP y TEP en pacientes sometidos a cirugía ortognática en el Hospital Clínico de la Pontificia Universidad Católica de Chile en el período transcurrido entre octubre de 2006 y enero de 2016.

\section{Pacientes y Métodos}

Se realizó un registro mediante la revisión de fichas clínicas de los pacientes sometidos a cirugía ortognática bajo anestesia general en el Hospital Clínico de la Pontificia Universidad Católica de Chile en el período comprendido entre octubre de 2006 y enero de 2016.

Se incorporaron en el estudio todos los pacientes sometidos a cirugía ortognática incluyendo osteotomías bimaxilares, osteotomías Le Fort I, osteotomía sagital de rama uni o bilateral y genioplastía.

Se realizó una revisión de las fichas clínicas hospitalarias y, además, se evaluaron los registros de controles posoperatorios en busca de historia de TEP o TVP, como dolor, aumento de volumen y eritema de extremidades inferiores, disnea y dolor torácico.

En los datos registrados se incluyó toda la información correspondiente a potenciales factores de riesgo de TEV (Tabla 1). Los pacientes fueron categorizados según la escala de estratificación de riesgo de Geerts ${ }^{3}$ (Tabla 2).

Para el registro de los datos se utilizó el programa Epi Info (Versión 7.0, CDC, Atlanta, USA) (Anexo 1). Los resultados fueron expresados en valores de frecuencia absoluta y relativa.

Tabla 1. Datos registrados

\begin{tabular}{|ll|}
\hline Género & Edad \\
\hline Diagnóstico & Tipo de cirugía \\
Fecha de ingreso & Duración de hospitalización \\
\hline Peso/Altura & IMC \\
Tabaquismo & Anticonceptivos orales \\
$\begin{array}{l}\text { Antecedentes mórbidos (diabetes, enfermedad } \\
\text { cardiovascular, tumores malignos) }\end{array}$ & Complicaciones de la cirugía \\
\hline
\end{tabular}


Tabla 2. Estratificación de riesgo de TEV en pacientes quirúrgicos sin profilaxis

\begin{tabular}{|c|c|c|c|c|c|}
\hline \multirow[b]{2}{*}{ Nivel de Riesgo } & \multirow{2}{*}{$\begin{array}{r}\text { TVP, } \% \\
\text { Distal }\end{array}$} & \multicolumn{3}{|c|}{ TEP, \% } & \multirow[t]{2}{*}{ Estrategias de prevención sugeridas } \\
\hline & & Proximal & Clínico & Fatal & \\
\hline $\begin{array}{l}\text { Bajo Riesgo } \\
\text { - Cirugía menor en pacientes menores de } 40 \text { años sin } \\
\text { otros factores de riesgo }\end{array}$ & 2 & 0,4 & 0,2 & $<0,01$ & $\begin{array}{l}\text { Sin profilaxis específica: movilización } \\
\text { temprana y "agresiva" }\end{array}$ \\
\hline $\begin{array}{l}\text { Riesgo Moderado } \\
\text { - Cirugía menor en pacientes con factores de riesgo } \\
\text { adicionales } \\
\text { - Cirugía en pacientes entre } 40-60 \text { años sin factores } \\
\text { de riesgo adicionales }\end{array}$ & $10-20$ & $2-4$ & $1-2$ & $0,1-0,4$ & $\begin{array}{l}\text { LDUH (q12h), LMWH } \\
(\leq 3.400 \text { U diaria), GCS or IPC }\end{array}$ \\
\hline $\begin{array}{l}\text { Alto Riesgo } \\
\text { - Cirugía en pacientes mayores de } 60 \text { años } \\
\text { - Cirugía en pacientes entre } 40 \text { y } 60 \text { años con fac- } \\
\text { tores de riesgo adicionales (TEV previo, cáncer, } \\
\text { estados de hipercoagulabilidad) }\end{array}$ & $20-40$ & $4-8$ & $2-4$ & $0,4-1,0$ & $\begin{array}{l}\text { LDUH (q8h), LMWH } \\
\text { (>3.400 U diaria), GCS or IPC }\end{array}$ \\
\hline $\begin{array}{l}\text { Más Alto Riesgo } \\
\text { - Cirugía en pacientes con múltiples factores de } \\
\text { riesgo } \\
\text { - Artroplastía de cadera o tobillo } \\
\text { - Trauma mayor }\end{array}$ & $40-80$ & $10-20$ & $4-10$ & $0,2-5$ & $\begin{array}{l}\text { LMWH (>3.400 U diaria), } \\
\text { fondaparinux, oral VKAs (INR 2-3), } \\
\text { or IPC/GCS + LDUH/LMWH }\end{array}$ \\
\hline
\end{tabular}

Modificado de Geerts y cols ${ }^{3}$. TEV: tromboembolismo venoso; TVP: trombosis venosa profunda; TEP: tromboembolismo pulmonar; LDUH: lowdose unfractioned heparin; LMWH: low molecular weight heparin; GCS: graduated compression stockings; IPC: intermittent pneumatic compression; VKA: vitamin $\mathrm{K}$ antagonist.

\section{Resultados}

Entre octubre de 2006 y enero de 2016 se realizaron 86 cirugías ortognáticas, de las cuales 35 se realizaron en hombres y 51 en mujeres. La muestra se clasificó según el tipo de cirugía: osteotomía maxilar, osteotomía mandibular y osteotomías bimaxilares. La edad de los pacientes, tiempo de cirugía y tiempo de hospitalización se resumen en la Tabla 3.

Todos los pacientes fueron estratificados según riesgo de TEV de acuerdo a la categorización de Geerts ${ }^{3}$, categorizándolos en bajo, moderado, alto o muy alto riesgo de TEV. De los 86 casos estudiados, ninguno fue clasificado en el grupo de muy alto riesgo y sólo 2 fueron considerados como pacientes de alto riesgo (Tabla 4).

De los datos recolectados sólo se identificaron dos pacientes que evolucionaron con un tromboembolismo pulmonar, lo cual representa al 2,3\% de los pacientes operados durante el período descrito. Los detalles se especifican en la Tabla 5.

El primer caso corresponde a una paciente de género femenino, 51 años de edad, con antecedentes de hipotiroidismo en tratamiento con levotiroxina. Se programó una osteotomía Le Fort I, osteotomía sagital de rama bilateral y genioplastía de avance, la cual duró $6 \mathrm{~h}$ aproximadamente.

Como medidas antitrombóticas se administró enoxaparina y se mantuvo con medias compresivas hasta iniciada la kinesioterapia motora, lo cual ocurrió al segundo día posoperatorio. La estadía hospitalaria fue de 5 días.

En el día 14 de su posoperatorio la paciente acude al Servicio de Urgencia del Hospital Clínico de la Pontificia Universidad Católica de Chile por un síncope, además de disnea de 5 días y palpitaciones. Fue hospitalizada luego de la confirmación diagnóstica de un TEP.

El segundo caso corresponde a un paciente de género masculino, 25 años de edad, con antecedente de tabaquismo. Fue sometido a una cirugía ortognática triple (osteotomía Le Fort I, osteotomía sagital de rama bilateral y genioplastía de avance), con una duración de cirugía de $6 \mathrm{~h}$. La tromboprofilaxis fue administrada de la misma manera que en el primer caso hasta comenzar la kinesioterapia motora, lo cual ocurrió en su segundo día posoperatorio. La estadía hospitalaria fue de 5 días.

En el día 11 de su posoperatorio consultó en el servicio de urgencia por dolor torácico derecho con tope inspiratorio de 2 días de evolución, sin fiebre ni disnea. El paciente fue evaluado mediante un angioTAC que confirmó el diagnóstico de TEP. 
Tabla 3. Datos obtenidos de cirugías ortognáticas registradas expresados en promedios (desviación estándar) exceptuando el $\mathbf{N}$ de pacientes

\begin{tabular}{|lcccc|}
\hline Cirugía & $\begin{array}{c}\text { N } \\
\text { (Hombre/Mujeres) }\end{array}$ & $\begin{array}{c}\text { Edad } \\
\text { (años) }\end{array}$ & $\begin{array}{c}\text { Duración de la cirugía } \\
\text { (horas) }\end{array}$ & $\begin{array}{c}\text { Duración de hospitalización } \\
\text { (días) }\end{array}$ \\
\hline Osteotomía maxilar & $10(2 / 8)$ & $26,7(8,8)$ & $3,1(1,3)$ & $3,4(0,8)$ \\
Osteotomía mandibular & $13 \quad(7 / 6)$ & $23 \quad(5,5)$ & $4(1,6)$ & $3,6(1,1)$ \\
Osteotomía bimaxilar & $63(26 / 37)$ & $25,5(9,8)$ & $5,6(1,5)$ & $4,5(1,6)$ \\
\hline
\end{tabular}

Tabla 4. Nivel de riesgo de TEV y tipo de cirugía

\begin{tabular}{|lcccc|}
\hline Cirugía & Bajo riesgo & Moderado riesgo & Alto riesgo & Muy alto riesgo \\
\hline Osteotomía maxilar & 4 & 6 & 0 & 0 \\
\hline Osteotomía mandibular & 7 & 6 & 0 & 0 \\
Osteotomía bimaxilar & 34 & 27 & 2 & 0 \\
\hline
\end{tabular}

Tabla 5. Datos extraídos de pacientes con TEV poscirugía ortognática

\begin{tabular}{|lll|}
\hline Sexo & Femenino & Masculino \\
\hline Edad (años) & 51 & 25 \\
\hline Rirugía & $\begin{array}{l}\text { Osteotomía Le Fort I, Osteotomía sagital bilateral } \\
\text { de rama mandibular, genioplastía }\end{array}$ & $\begin{array}{l}\text { Osteotomía Le Fort I, Osteotomía sagital bilateral } \\
\text { de rama mandibular, genioplastía }\end{array}$ \\
\hline Profilaxis antitrombótica & Moderado riesgo & Bajo riesgo \\
\hline Duración de la cirugía (horas) & Sí & Sí \\
\hline Duración de la hospitalización (días) & 5 & 6 \\
\hline Duración de la inmovilización (días) & 2 & 5 \\
\hline
\end{tabular}

\section{Discusión}

Actualmente existen datos muy limitados respecto a la incidencia de TVP y TEP en cirugía maxilofacial, sin embargo se ha demostrado que la incidencia de TEV es excepcionalmente baja, variando entre un $0,15-1,6 \%$. La mayoría de los pacientes que sufren un evento tromboembólico tienen antecedentes de neoplasias, trauma mayor y cirugías prolongadas como principales factores de riesgo.

Dos estudios realizados en $V U$ University $M e$ dical Center de Ámsterdam evaluaron la incidencia de TEV en pacientes que fueron sometidos a procedimientos de cirugía maxilofacial sin aplicar medidas tromboprofilácticas. El primero registró una incidencia de $0,2 \%$ en pacientes operados por trauma facial ${ }^{5}$, mientras que el segundo registró una incidencia de $0,5 \%$ para pacientes sometidos a otros procedimientos de cirugía maxilofacial tales como cirugía dentoalveolar, distracción osteogénica, quistectomías, escisiones tumorales, parotidectomía, inserción de implantes, cirugía preimplantaria, cirugía reconstructiva, fisurados, cirugía de articulación temporomandibular, retiro de material de osteosíntesis y cirugía ortognática ${ }^{6}$.

Verlinden y cols $^{2}$ analizaron en forma retrospectiva una cohorte de 4.127 pacientes sometidos a cirugía ortognática o distracción osteogénica de los maxilares (considerando pacientes sometidos a osteotomías mandibulares, osteotomías maxilares, ambas juntas o distracciones osteogénicas) sin uso de medidas tromboprofilácticas, identificando solo dos casos de TVP (de los cuales uno evolucionó a TEP). Los autores justifican esta baja incidencia en que los pacientes de cirugía ortognática por lo general comienzan a movilizarse en forma temprana 
y se les da un alta precoz, disminuyendo tiempos de inmovilización y, por lo tanto, el riesgo de TEV. Por otra parte, Blackburn y cols ${ }^{7}$, describieron 2 casos en una cohorte retrospectiva de 127 pacientes sometidos a cirugía ortognática, uno de los cuales había sido sometido a un autoinjerto de cresta ilíaca.

Incidencias de este orden situarían a la cirugía maxilofacial como una disciplina en la que nos enfrentamos a una situación de bajo riesgo de TEV, lo cual hace cuestionarse si es costo-efectivo aplicar medidas de tromboprofilaxis a todos los pacientes, más aún si consideramos que algunas de estas medidas (principalmente farmacológicas) pueden aumentar el riesgo de hemorragia posoperatoria.

En la cohorte analizada del presente estudio, sólo se consideraron pacientes sometidos a cirugía ortognática. La mayor incidencia reportada en este estudio respecto a lo descrito a la literatura podría explicarse por tiempo operatorio prolongado, como factor de riesgo a desarrollar un TEV.

En relación a la cirugía maxilofacial, Skorpil y cols ${ }^{5}$ sólo encontraron una relación estadísticamente significativa entre tiempo de cirugía y riesgo de trombosis, mientras que en los pacientes sometidos a cirugía electiva, incluidos en el estudio de Forouzanfar y cols ${ }^{6}$, se encontró una relación de riesgo en pacientes que fueron sometidos a autoinjertos óseos obtenidos de cresta iliaca y TEV.

Esta situación ha determinado que no haya una aplicación de medidas de tromboprofilaxis ampliamente utilizada. Una encuesta realizada en el Reino Unido a cirujanos orales y maxilofaciales reveló que un $67 \%$ de ellos utilizaba medidas tromboprofilácticas de manera rutinaria, 14\% las utilizaba en forma ocasional y $19 \%$ no tomaban medidas tromboprofilácticas; la mayoría de los cirujanos que no utilizaban medidas antitrombóticas justificaban dicha conducta por la baja incidencia de TEV en cirugía maxilofacial ${ }^{8}$.

Si bien no hay un consenso universal respecto a la valoración de riesgo de estos pacientes, se han propuesto varios scores que permiten evaluar el riesgo de padecer alguna de estas complicaciones en el período perioperatorio y durante la estadía hospitalaria. Además, hay una serie de factores reconocidos ampliamente en la literatura como de alto y bajo riesgo. Es por esto que es de suma importancia reconocer dichos factores en los pacientes candidatos a ser sometidos a procedimientos quirúrgicos y aplicar las medidas profilácticas correspondientes ${ }^{9}$. En nuestro Centro los pacientes son categorizados de acuerdo a la escala de riesgo de TEV de Caprini (Tabla 6).

Algunos autores como Comerota y cols ${ }^{10}$ han propuesto que el principal mecanismo de acción de los métodos tromboprofilácticos mecánicos es evitar la venodilatación estimulada por la inmovilización y la anestesia general. Las medidas farmacológicas, por su parte, buscan revertir el estado de hipercoagulabilidad del paciente mediante el uso de fármacos anticoagulantes.

La heparina de bajo peso molecular presenta varias ventajas como agente tromboprofiláctico sobre otros agentes; no requiere monitorización mediante pruebas de laboratorio (TTPa) y tiene menores probabilidades de provocar trombocitopenia $\mathrm{u}$ osteoporosis. Las guías clínicas del American College of Chest Physicians ${ }^{3}$ recomiendan que en pacientes de bajo riesgo no se utilicen medidas tromboprofilácticas específicas aparte del uso de métodos mecánicos, mientras que en pacientes con mayor riesgo se recomienda el uso de tromboprofilaxis farmacológica.

Respecto al tiempo de tratamiento tromboprofiláctico, se debe prolongar más allá del período de hospitalización, ya que se ha descrito que puede haber complicaciones tromboembólicas tardías. De hecho, aproximadamente un tercio de los pacientes que desarrollan un TEV lo hacen entre 14 y 35 días posoperatorios $^{11}$. Esto puede relacionarse con los dos casos presentados en este artículo, los cuales desarrollaron un TEP en forma tardía y posterior al alta hospitalaria, a pesar de medidas aplicadas durante la hospitalización.

En nuestro Centro las medidas tromboprofilácticas, utilizadas como protocolo en pacientes sometidos a cirugía ortognática, son la administración de $40 \mathrm{mg}$ al día de enoxaparina en forma subcutánea hasta iniciada la movilización y medidas mecánicas (uso de medidas antitrombóticas y sistemas neumáticos de compresión en el intraoperatorio), además, de kinesioterapia motora del paciente en forma temprana (habitualmente desde el segundo día posoperatorio).

Muchos de los episodios de TVP pueden ser asintomáticos, pudiendo retrasar el diagnóstico hasta etapas más avanzadas en las que ya se presentan complicaciones como el TEP. Por otra parte, los episodios asintomáticos pueden resolverse de manera espontánea mediante la lisis del trombo producido, no siendo detectados y por lo tanto, subestimada la real incidencia de $\mathrm{TEV}^{3}$.

Los principales signos asociados a una TVP son edema asimétrico de extremidades inferiores, venas palpables induradas, pérdida de pulso periférico, fiebre y malestar general junto con eritema y aumento de calor local de la zona afectada. Cuando el cuadro progresa a un TEP puede presentarse con dolor torácico, disnea, desaturación de oxígeno, taquip- 
Tabla 6. Escala de valoración de riesgo de TVP de Caprini

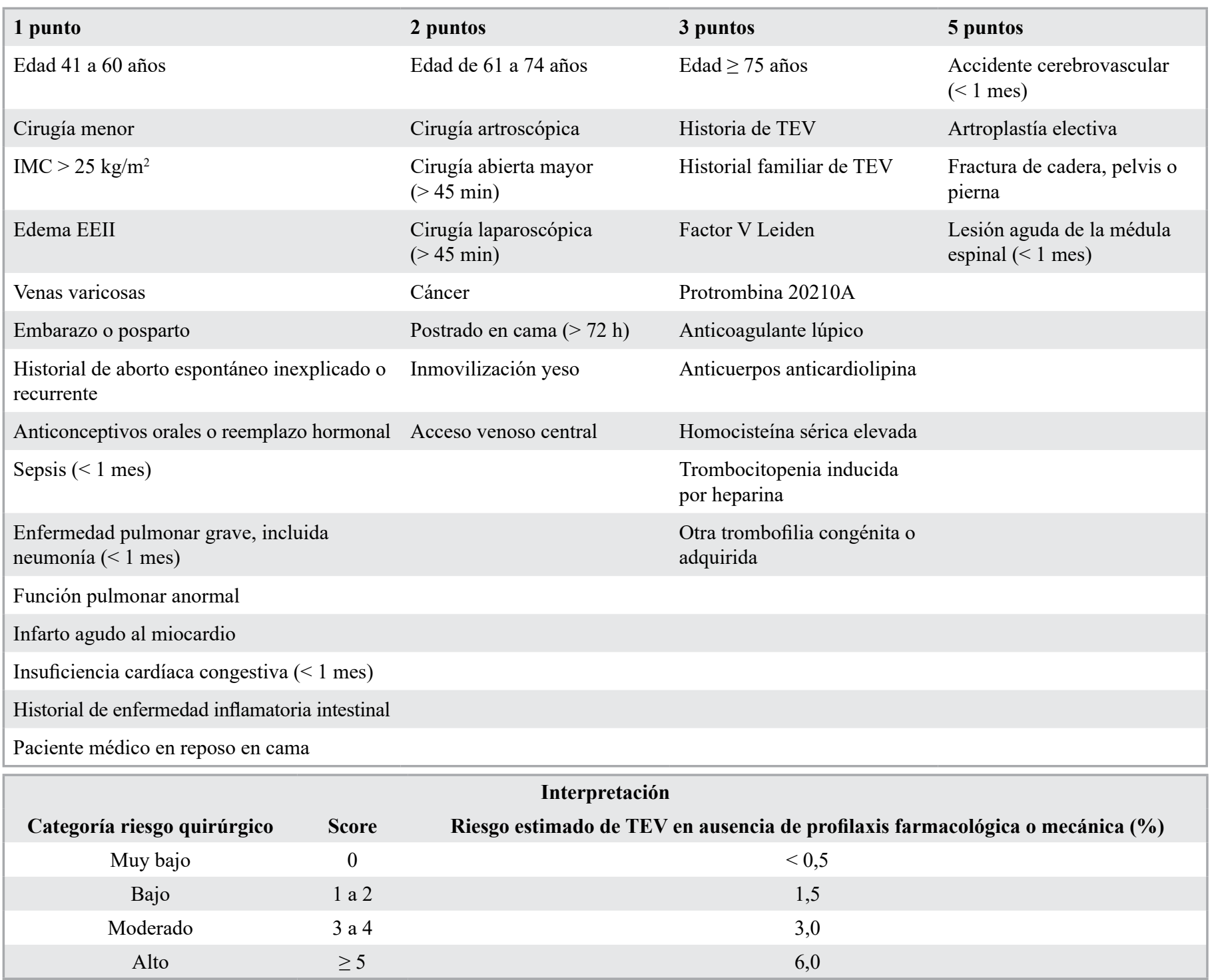

nea, taquicardia sinusal y hemoptisis ${ }^{9}$. Si bien en el segundo caso de TEP descrito en este estudio no presentaba mayor sintomatología, además de dolor torácico, podemos ver en el primer caso descrito que hubo una mayor sintomatología asociada, incluyendo disnea progresiva, palpitaciones, taquicardia y un síncope. Esta variabilidad en la sintomatología presentada nos habla de la diversidad de la presentación clínica de la enfermedad. Además, ninguno de los dos pacientes presentó signos clínicos de TVP previo al episodio de TEP, lo cual muestra que, si bien la TVP ocurre previo al TEP, ésta pudiera pasar desapercibida. En vista a ello y a la progresión que puede cursar el cuadro es que se debe estar alerta a los signos de TEV con los que pueden cursar los pacientes quirúrgicos, sobre todo ante la presencia de algún factor predictor de riesgo.

$\mathrm{Si}$ bien las limitaciones del presente estudio no permiten realizar un análisis estadístico de los principales factores de riesgo asociados a la ocurrencia de un TEV en pacientes sometidos a cirugía ortognática, se pudo ver una incidencia de un $2,6 \%$ en un total de 86 pacientes. Aunque la literatura describe una baja incidencia de TEV en pacientes sometidos 
a cirugía maxilofacial y el uso de métodos profilácticos farmacológicos es menos usado dada la incidencia excepcionalmente baja de TEP y TVP descrito en este tipo de cirugía, la magnitud de las complicaciones propias del cuadro y su progresión hace que se deba valorar en forma adecuada el manejo profiláctico de dichos pacientes.

Es fundamental considerar el riesgo individual del paciente y el riesgo que implica la cirugía. Si bien la cirugía ortognática no es considerada una cirugía de alto riesgo de $\mathrm{TEV}$, la prolongación del tiempo quirúrgico, de la estadía hospitalaria y la inmovilización del paciente pueden determinar un riesgo mayor de desarrollar una complicación de este tipo.

\section{Responsabilidades éticas}

Protección de personas y animales. Los autores declaran que para esta investigación no se han realizado experimentos en seres humanos ni en animales.

Confidencialidad de los datos. Los autores declaran que en este artículo no aparecen datos de pacientes.

Derecho a la privacidad y consentimiento informado. Los autores declaran que en este artículo no aparecen datos de pacientes.

Financiamiento: Sin financiamiento.

Conflicto de Intereses: No hay.

\section{Referencias}

1. Anderson FA, Spencer FA. Risk factors for venous thromboembolism. Circulation 2003;107(23 Suppl. 1):19-26.

2. Verlinden CRA, Tuinzing DB, Forouzanfar T. Symptomatic venous thromboembolism in orthognathic surgery and distraction osteogenesis: A retrospective cohort study of 4127 patients. Br J Oral Maxillofac Surg. 2014;52:401-4.

3. Geerts WH, Pineo GF, Heit JA, Bergqvist D, Lassen MR, Colwell CW. Thromboembolism The Seventh ACCP Conference on Antithrombotic and Thrombolytic. Chest 2004;126:338s-400s.

4. Heit JA. Venous thromboembolism: Disease burden, outcomes and risk factors. J Thromb Haemost. 2005;3:16117.

5. Skorpil N, Van Den Bergh B, Heymans MW, Forouzanfar T. Is thromboembolism prophylaxis necessary for low and moderate risk patients in maxillofacial trauma? A retrospective analysis. Int J Oral Maxillofac Surg. 2012;41:902-5.

6. Forouzanfar T, Heymans MW, van Schuilenburg A, Zweegman $\mathrm{S}$, Schulten EAJM. Incidence of venous thromboembolism in oral and maxillofacial surgery: a retrospective analysis. Int J Oral Maxillofac Surg. 2010;39:256-9.

7. Blackburn TK, Pritchard K, Richardson D. Symptomatic venous thromboembolism after orthognathic operations: An audit. $\mathrm{Br}$ J Oral Maxillofac Surg. 2006;44:389-92.

8. Farr DR, Hare AR. The use of thromboembolic prophylaxis in oral and maxillofacial surgery. Br J Oral Maxillofac Surg. 1994;32:161-4.

9. Williams B, Indresano AT, Ryan FO. Venous Thromboembolism in Oral and Maxillofacial Surgery: A Review of the Literature. Yjoms 2011;69:840-4

10. Comerota AJ, Stewart GJ, Alburger PD, Smalley K, White JV. Operative venodilation: a previously unsuspected factor in the cause of postoperative deep vein thrombosis. Surgery 1989;106:301-9.

11. Dahl OE, Andreassen G, Aspelin T, Muller C, Mathiesen P, Nyhus S, et al. Prolonged thromboprophylaxis following hip replacement surgery-results of a double-blind, prospective, randomised, placebo-controlled study with dalteparin
(Fragmin). Thromb Haemost. 1997;77:2631.

12. Zhan C, Miller MR. Excess Length of Stay, Charges, and Mortality Attributable to Medical Injuries During Hospitalization. JAMA 2003;290:186874.

13. Heit JA. Venous thromboembolism: Disease burden, outcomes and risk factors. J Thromb Haemost. 2005;3:1611-7.

14. Prandoni P, Samama MM. Risk stratification and venous thromboprophylaxis in hospitalized medical and cancer patients. Br J Haematol. 2008;141:587-97.

15. Coleridge-Smith PD, Hasty JH, Scurr JH. Venous stasis and vein lumen changes during surgery. Br J Surg. 1990;77:10559.

16. Hull RD, Hirsh J, Sackett DL, Stoddart GL. Cost-effectiveness of primary and secondary prevention of fatal pulmonary embolism in high-risk surgical patients. Can Med Assoc J. 1982;127:990-3.

17. Passman MA. Mandated quality measures and economic implications of venous thromboembolism prevention and management. Am J Surg. 2010;199(1 Suppl):S21-31. 